\title{
Shock dynamics induced by double-spot laser irradiation of layered targets
}

\author{
Abutrab A. Aliverdiev, \\ Dimitri Batani, \\ Anise A. Amirova, \\ Roberto Benocci, \\ Riccardo Dezulian, \\ Eduard Krouský, \\ Miroslav Pfeifer, \\ Jiři Skala, \\ Roman Dudzak, \\ Katarzyna Jakubowska
}

\begin{abstract}
We studied the interaction of a double-spot laser beam with targets using the Prague Asterix Laser System (PALS) iodine laser working at $0.44 \mu \mathrm{m}$ wavelength and intensity of about $10^{15} \mathrm{~W} / \mathrm{cm}^{2}$. Shock breakout signals were recorder using time-resolved self-emission from target rear side of irradiated targets. We compared the behavior of pure $\mathrm{Al}$ targets and of targets with a foam layer on the laser side. Results have been simulated using hydrodynamic numerical codes.
\end{abstract}

Key words: laser shock $\bullet$ high pressures $\bullet$ non-uniformity smoothing

\author{
A. A. Aliverdiev \\ Institute for Geothermal Researches of Dagestan \\ Scientific Center of Russian Academy of Sciences, \\ 30A Shamilya Pr., 367030, Makhachkala, Russia, \\ Tel.: +7 9285603898 , \\ E-mail: aliverdi@mail.ru

\section{Batani} \\ University Bordeaux, \\ CEA, CNRS, CELIA (Centre Laser Intense \\ at Applications), \\ UMR 5107, F-33405 Talence, France
}

\section{A. A. Amirova}

Institute of Physics of Dagestan Scientific Center of Russian Academy of Sciences,

94 Yaragskogo Str., 367003, Makhachkala, Russia

R. Benocci, R. Dezulian

Dipartimento di Fisica "G. Occhialini",

Università di Milano Bicocca,

Milan, Italy

E. Krouský, M. Pfeifer, J. Skala, R. Dudzak

PALS Research Centre,

Za Slovankou 3, 18221 Prague 8, Czech Republic

\section{K. Jakubowska}

Institute of Plasma Physics and Laser Microfusion, 23 Hery Str., 01-497 Warsaw, Poland and University Bordeaux,

CEA, CNRS, CELIA (Centre Laser Intense at Applications),

UMR 5107, F-33405 Talence, France

Received: 11 July 2014

Accepted: 19 December 2014

\section{Introduction}

It is well known that laser-driven inertial confinement fusion (ICF) as well as the investigation of laser-driven extreme states of matter require a very high degree of uniformity of laser irradiation. Smoothing techniques (such as phased zone plates [1-3], random phase plates [4], kinoform phase plates [5], smoothing by spectral dispersion [6], induced spatial incoherence [7]) have dramatically improved our control on laser implosions and laser-plasma interactions. However, there still remains an issue of non-uniformity at very early times, called 'laser imprint' problem [8-12], which may affect compression uniformity at later times and in particular influence the development of the Rayleigh-Taylor instability [13]. In this context, low-density foams can be used both as a mean of shock pressure amplification due to impedance mismatch effect [14] and as means of producing uniform energy deposition [15]. In order to analyze the possibility of smoothing large-scale non-uniformities using low density foams we have realized series of experiments, where the large-scale non-uniform irradiation was set by splitting the laser beam in two equal parts with a prism and producing a double focal spot on the target. This experiment follows the experimental arrangement used in Ref. [16] where a gas layer (produced through a gas jet) in front of the target was used to smooth the non-uniformities of the laser irradiation. However, in the present case, the presence of the foam layer before the payload target ( $\mathrm{Al}$ in our 
case), introduced some peculiar behavior in shock dynamics. Namely, we obtained evidence of collision between the two shocks originating from each of the two spots. When this is the case, the presence of the foam does not necessarily smooth out the large-scale laser non-uniformities, but may instead induce a higher local pressure, which would of course be deleterious for ICF (small-scale non-uniformities will be smoothed out more rapidly and therefore they would not produce effects of this type). Indeed the goal of the present paper is to analyze the peculiarity of the dynamics and the interaction of the two shocks generated by a double focal spot.

\section{Experiment}

Experiments were realized using the PALS (Prague Asterix Laser System) iodine laser [17]. The laser pulse at $0.44 \mu \mathrm{m}$ (the third harmonic of the emission wavelength) was Gaussian in time with a full width at half maximum (FWHM) of 400 ps and a single-shot energy up to $400 \mathrm{~J}$. We used the same experimental set-up and diagnostics system as described in Ref. [16]. By splitting the laser beam in two equal parts with a prism we could obtain two focal spots, each with a diameter of about $30-100 \mu \mathrm{m}$, at a separation of $100-200 \mu \mathrm{m}$, thus producing a very large-scale irradiation non-uniformity. As for the diagnostics, we used time-resolved self-emission for the detection of the shock breakout at the target rear face. A photographic objective was employed for imaging of the target rear surface onto a streak camera (Hamamatsu C7700 with S-1 photocathode). A red RG60 filter before the streak camera was used to cut out any $3 \omega$ light. The spatial resolution of the diagnostic was $2.6 \mu \mathrm{m}$ and the temporal resolution was 3.12 ps.

The targets used in the experiment were either simple Al foils ( $10 \mu \mathrm{m}$ thick) or double-layer targets made of foam (50 $\mu \mathrm{m}$ thick) and $\mathrm{Al}(10 \mu \mathrm{m}$ thick).

One of the main experimental results is the difference in time-resolved rear-side self-emission images obtained with the streak camera for aluminum and aluminum-foam targets (see Fig. 1). If in the first case (Al target) the two shocks generated by the two separated laser spots emerge separately on target rear side. In the second case $(\mathrm{Al}+$ foam target) the shock breakouts are quite larger and the two emission regions merge with each other, so that the central part becomes brighter than the regions corresponding to the positions of the two focal spots. The larger spatial shape of the shock breakout is due to both the longest target thickness (producing a larger 2D effect during shock propagation) and to a smoothing effect, i.e. the fact that due to the low density of the foam (i.e. longer electron mean free path) and large thickness of the created plasma the electron energy transport is more isotropic, i.e. a homogenization of energy deposition occurs. The appearance of the brighter region in between the two spots is instead due to the collisions of the two shocks, and this paper is indeed focusing on the analysis of such effect.

\section{Analysis and simulations}

The dependence of shock characteristics on the foam thickness and the pulse energy is well described by a simple hydrodynamic model of shock reverberation from the foam-Al interface [14]. With the assumption of the ideal gas equation of state both for the aluminum and the foam, their shock polar in the pressure-fluid velocity plane can be written as:

$$
P=\frac{\gamma+1}{2} \rho \cdot u^{2}
$$

where $u$ is the fluid velocity, $\rho$ is the density of considered material, and $\gamma$ is the adiabatic exponent (for a monoatomic perfect gas $\gamma=5 / 3$ ).

The shock velocity in this case is given by:

$$
D=\left(\left(\frac{\gamma+1}{2}\right)\left(\frac{P}{\rho}\right)\right)^{1 / 2}
$$

The impedance of aluminum is much higher than the impedance of foam, so after the reflection of the shock from the interface two shock waves are generated, one is transmitted to the aluminum layer and the other reflected back into the foam [18]. By assuming that the curve for the reflected shock into the foam is simply the mirror curve of the principal Hugoniot of the foam (an assumption which is well satisfied for weak shocks [18]) and by assuming that the shock reaches the interface when it is already stationary we can calculate the pressure in aluminum $P_{\mathrm{Al}}$ as [14]:

$$
P_{\mathrm{Al}}=\frac{4 \rho_{\mathrm{Al}}^{0}\left(\gamma_{\mathrm{Al}}+1\right)}{\left(\sqrt{\gamma_{\text {foam }}+1} \sqrt{\rho_{\text {foam }}}+\sqrt{\gamma_{\mathrm{Al}}+1} \sqrt{\rho_{\mathrm{Al}}^{0}}\right)^{2}} P_{a b l}
$$

where $\rho_{\mathrm{Al}}^{0}$ and $\rho_{\text {foam }}^{0}$ indicate the unperturbed foam and metal density, respectively, and $P_{a b l}$ is the ablation pressure, which can be estimated as [19]:

$$
P_{a b l} \approx 8.6\left(I / 10^{14}\right)^{2 / 3} \lambda^{-2 / 3}(A / 2 Z)^{1 / 3}
$$

where $I$ is the laser intensity on the target in $\mathrm{W} / \mathrm{cm}^{2}$, which can be calculated from the actual pulse energy and spot diameter, $\lambda$ is the laser wavelength in $\mu \mathrm{m}$, and $A, Z$ are the mass number and the atomic number, respectively.

Figure 1 presents rear-side optical streak camera images for shots with identical parameters (including laser energy), but aimed at different targets: 1) $10 \mu \mathrm{m} \mathrm{Al}$ (left), and 2) $10 \mu \mathrm{m} \mathrm{Al}+50 \mu \mathrm{m}$ foam (right). The main features of the image on the right are:

- the shock breakout appears to be larger;

- the shock breakout is delayed, with respect to the image on the left;

- there is a bright region between the two spots, which is absent in the case of the target without the foam ablator.

All these effects were reproducible in other shots.

Clearly, the experiment cannot be explained in 1D. In reality the geometry of the experiment, with the double-spot arrangement, is intrinsically 3D. However, in order to provide a preliminary analysis we have realized 2D simulations with the hydrocode 

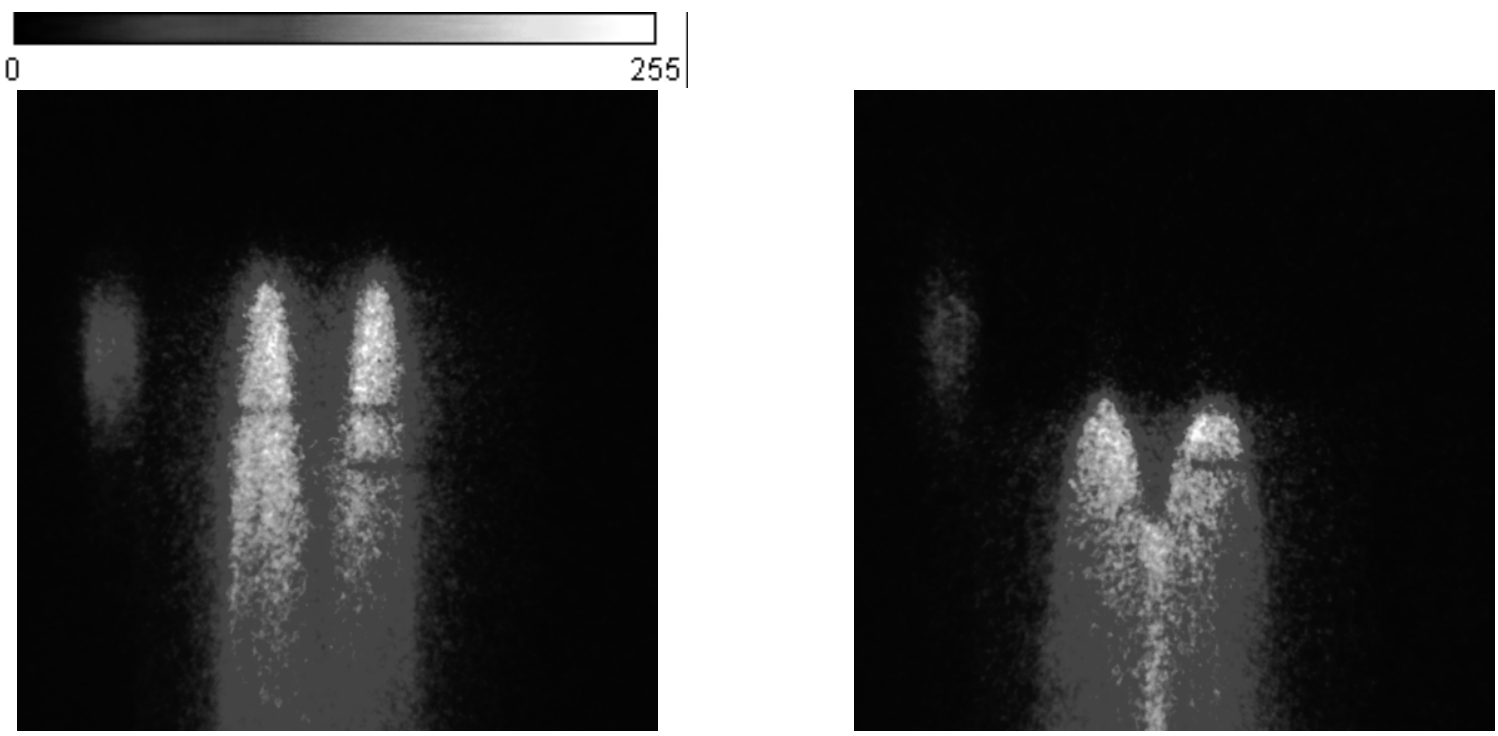

Fig. 1. Rear-side optical streak camera images: 1) (left) $10 \mu \mathrm{m} \mathrm{Al}$ target (shot 141), 2) (right) target with $10 \mu \mathrm{m} \mathrm{Al}$ $+50 \mu \mathrm{m}$ foam $\left(\rho_{\text {foam }}=50 \mathrm{mg} / \mathrm{cm}^{3}\right.$, shot 148 ). The total time window is $1600 \mathrm{ps}$ (vertical) and the imaged region is $1330 \mu \mathrm{m}$ wide (horizontal). The laser energy is the same for both shots: $E=113 \mathrm{~J}$. Time flows from top to bottom. The signal on the upper right part of the image is the time fiducial indicating the arrival of the laser pulse on the front side of the target.

MULTI [20], which we recently used in analyzing and interpreting several experimental results related to laser-produced plasmas [21] and extreme states of matter [22]. In order to simulate the experiment with a $2 \mathrm{D}$ code, we assumed a large non-uniformity in axial-symmetric approximation, i.e. we have used a ring spatial profile for the laser spot. Although this is not a completely realistic model for the considered experiment, nevertheless it gives the possibility for at least a preliminary qualitative analysis, and provides a base for further modeling.

Figure 2 shows the pressure profiles for the $\mathrm{Al}$ (upper) and the foam-Al (lower) targets at the time of shocks arrival to the target rear-side. The images on the left correspond to the time at which the shocks break out in correspondence with the center of the a

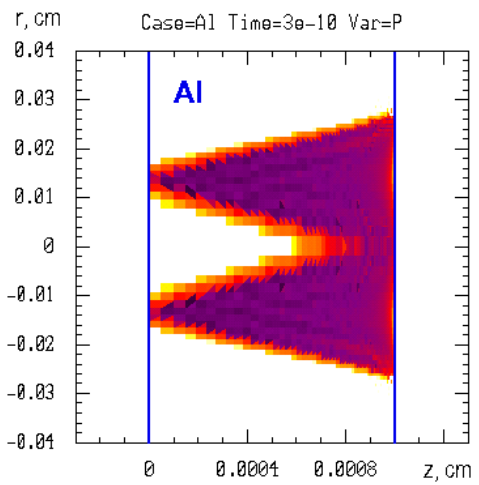

$\mathrm{r}, \mathrm{cm} \quad \mathrm{Cas} \theta=\mathrm{Al}-$ foam Time $=4.6 \theta-10$ Var $=\mathrm{P}$

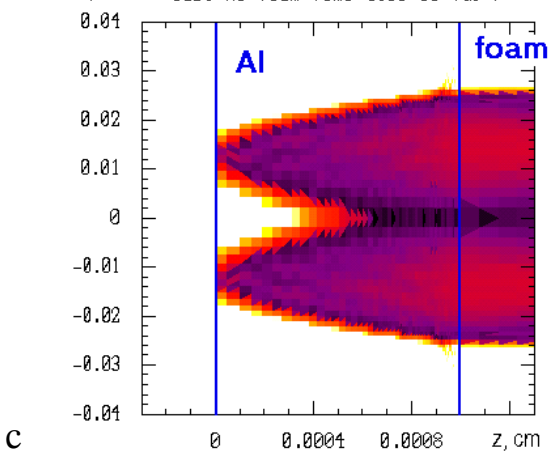

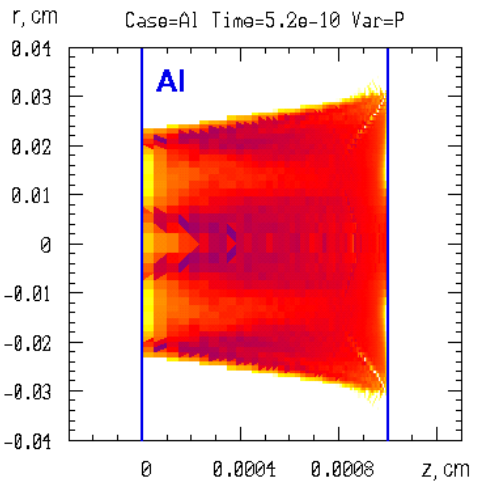

$\mathrm{P}(\mathrm{Mb})$

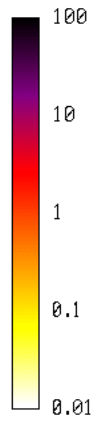

$\mathrm{r}, \mathrm{cm} \quad \mathrm{Cas} \theta=\mathrm{Al}-\mathrm{foam} \operatorname{Tim} \theta=6 \theta-10$ Var $=\mathrm{P}$
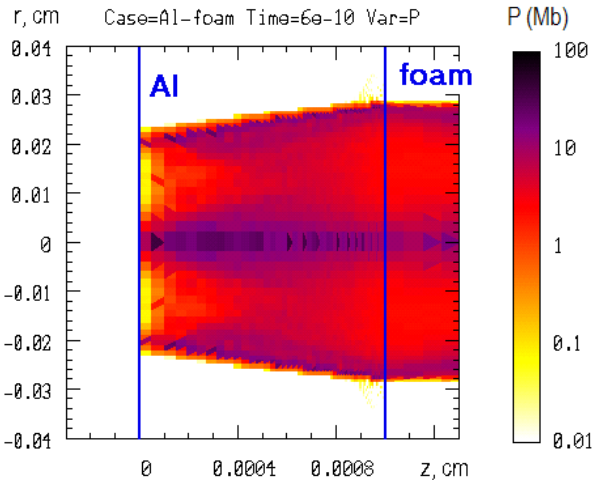

Fig. 2. Pressure profiles for $\mathrm{Al}$ (upper row) and Al-foam $\left(\rho_{\text {foam }}=50 \mathrm{mg} / \mathrm{cm}^{3}\right.$, lower row) targets in Lagrangian coordinates (only the Al layer is shown). We used an 'annular' laser profile (annulus central diameter $300 \mu \mathrm{m}$, and a Gaussian profile with FWHM $50 \mu \mathrm{m}$ ), the total laser power $P=0.2 \mathrm{TW}$, the laser wavelength $\lambda=0.44 \mu \mathrm{m}$, a Gaussian temporal profile with the FWHM duration $\tau=400$ ps. The laser beam comes from right. The images correspond to the time of the shock breakout (on the left) and to some later time (on the right). 

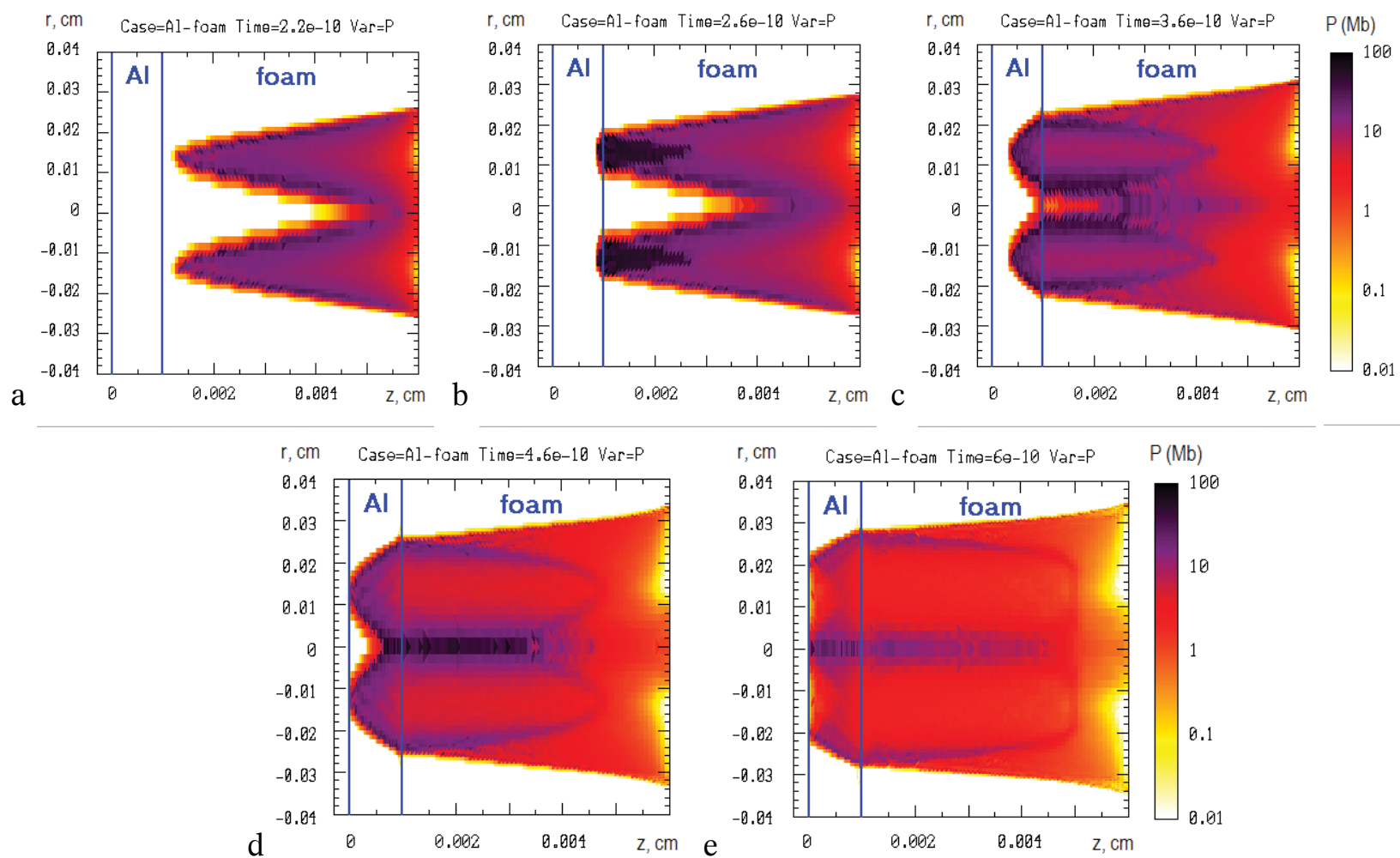

Fig. 3. The pressure profiles for the Al-foam $\left(\rho_{\mathrm{foam}}=50 \mathrm{mg} / \mathrm{cm}^{3}\right)$ targets in Lagrangian coordinates. The laser parameters are the same as in Fig. 2. a) The shock travels in the foam $\left(P_{\text {shock }} \approx 20\right.$ Mbar $)$. b) The shock reaches the Al-foam interface and is enhanced due to the impedance mismatch effect $\left(P_{\text {shock }} \approx 55 \mathrm{Mbar}\right)$. c) The shock travels in the $\mathrm{Al}$ layer and the reflected shock travels backward in the foam. d) The shock reaches the rear side of the Al layer. e) The shock reaches the rear side of the Al layer in the central region. The reflected shock still travels backward in the foam. A relaxation wave originating from the rear side of the Al layer travels backward into the Al.
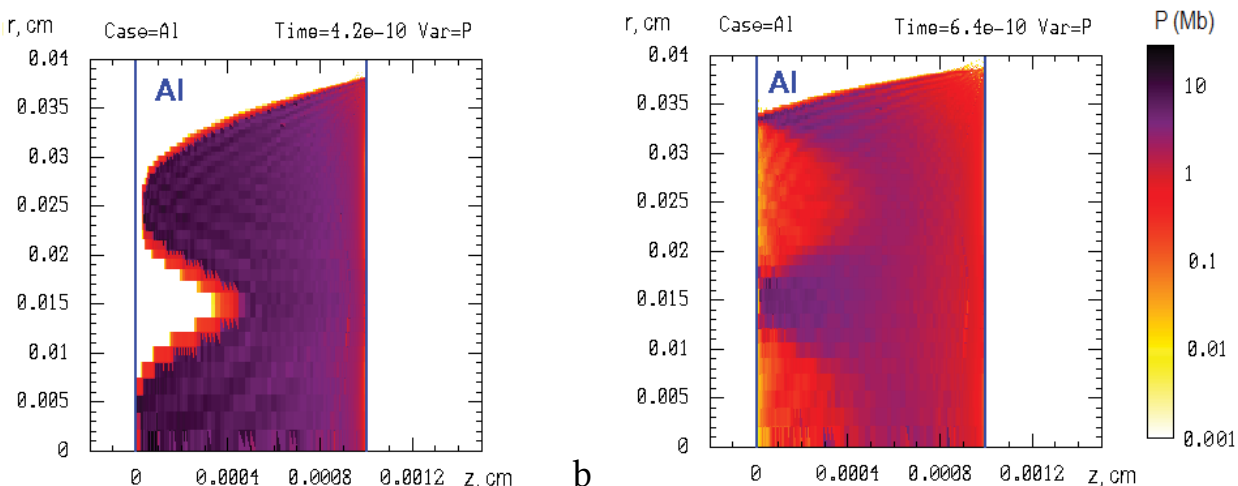

a
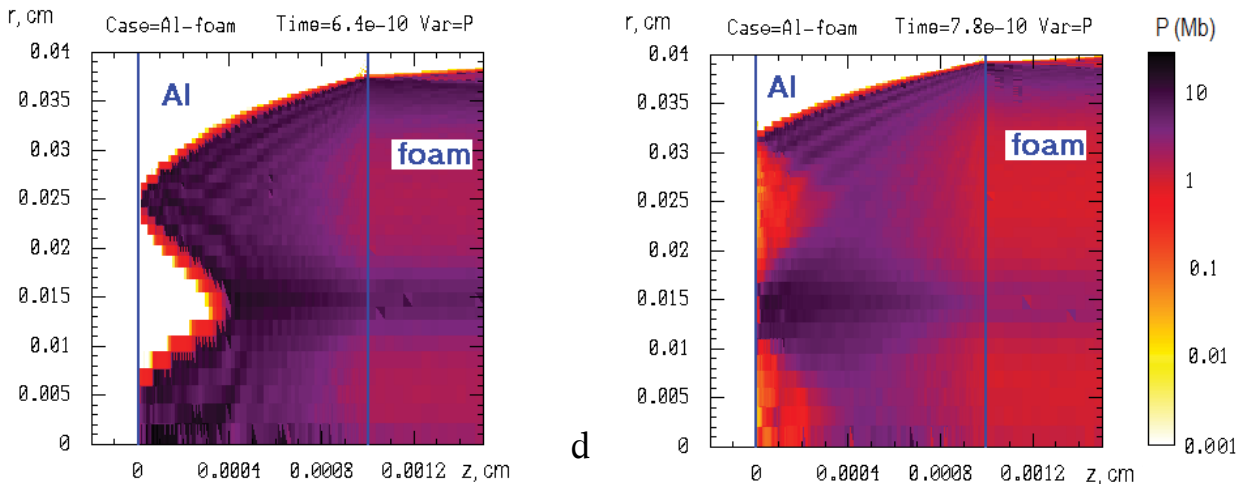

Fig. 4. Pressure profiles for $\mathrm{Al}$ (upper row) and Al-foam ( $\rho_{\text {foam }}=50 \mathrm{mg} / \mathrm{cm}^{3}$, lower row) targets in Lagrangian coordinates. We used a focal spot with a central circle and an annular structure around it (the central circle diameter $200 \mu \mathrm{m}$ and a planar ring with the internal and external diameters $400 \mu \mathrm{m}$ and $600 \mu \mathrm{m}$, respectively). The total laser power is $P=0.2 \mathrm{TW}$, the laser wavelength $\lambda=0.44 \mu \mathrm{m}$, a Gaussian temporal profile with the FWHM duration $\tau=400 \mathrm{ps}$. The laser beams comes from the right. In distinction to Fig. 2 here we present only half of the cylindrical target. 
laser spots. The image on the right corresponds to the time at which the shock breakout reaches the center between the two spots (i.e. the center of the ring in our $2 \mathrm{D}$ simulations)

In agreement with Eq. (4), which shows practically no dependence on the target material, the ablation pressures for both targets are close each other (the difference is less than few percent). The shock pressure in the $\mathrm{Al}$ layer of the double-layer target is increased due to impedance mismatch effect according Eq. (3). According to Eq. (3) for the chosen densities of foam $\left(50 \mathrm{mg} / \mathrm{cm}^{3}\right)$ and aluminum $\left(2.7 \mathrm{~g} / \mathrm{cm}^{3}\right)$, and using the approximation of an ideal monoatomic gas, the increase is approximately by a factor of 3.1. The $2 \mathrm{D}$ simulations with the tabular equations of state for both materials give an increase approximately by a factor of $2.5 \div 3$, in fair agreement with the simple analytical model. So the shock pressure in the foam-Al target is increased from 20 MBar up to 55 MBar for the considered case.

Also, the layered foam-Al targets clearly show a different behavior from simple Al foils. For the explanation let's see Fig. 3, which presents the pressure profiles within the full target size at different times. We see that in the simple Al foil, the region on the target rear side occupied by the initial shock breakout is quite small. Also, after the shock breakout, the reflected rarefaction wave damps the pressure. In contrast, in the Al-foam layered targets, the shock breakout is not only larger but the whole shock front inside the target material is larger. The two shocks originating from the two spots collide in the central region already before the shock breakout on the target rear side, which results in an increase of pressure. Another reason for the increase of pressure is the impedance mismatch at the foam-Al interface, as described by Eq. (3). The shock generated at the interface spreads back into the foam and continues to compress the foam-base. These phenomena produce a central region with a much higher pressure, as compared to the case of the simple Al foils, which in turn results in higher temperatures and higher emissivity in the center.

As we specified before, the $2 \mathrm{D}$ simulations with an axial symmetry are not completely adapted to reproduce our experiment. Indeed, the convergence of the shocks towards the axis can cause an artificial increase in pressure. Also the region around the center of the cylinder is critical for possible artefacts in discretization. Hence, in order to acquire more confidence in our results, we realized simulations with another spatial profile, i.e. we used a central circle with an annular structure around it. In this case indeed both the discretization problem and the problem of geometrical convergence are reduced, although they are not eliminated completely. A typical result of such a simulation is presented in Fig. 4, and, as we can see, the shock behavior is similar to that in the previous case. Although for the present simulation the direct shocks from the two spots have had time for their own collision even in the case of simple $\mathrm{Al}$ foils, which results in a small central peak (look at Fig. 4b), nevertheless the effect of the collision of reflected shocks is also clear (look at Fig. 4c-d). In general, the choice of the laser spot geometry is very important both for the simulations and for the experiment, and this will be the topic for the further work.

We should make a further comment on the comparison of our simulation results with the experimental streak camera images. The simulations can show the pressure dependence for the rear side (the last cell of the target) vs. time. Alternatively one can easily obtain figures showing the temperature dependence for the rear side vs. time (as presented in Figs. 5 and 6). Indeed, streak camera images give the emissivity of the target rear side in a spectral range corresponding to the sensitivity window of
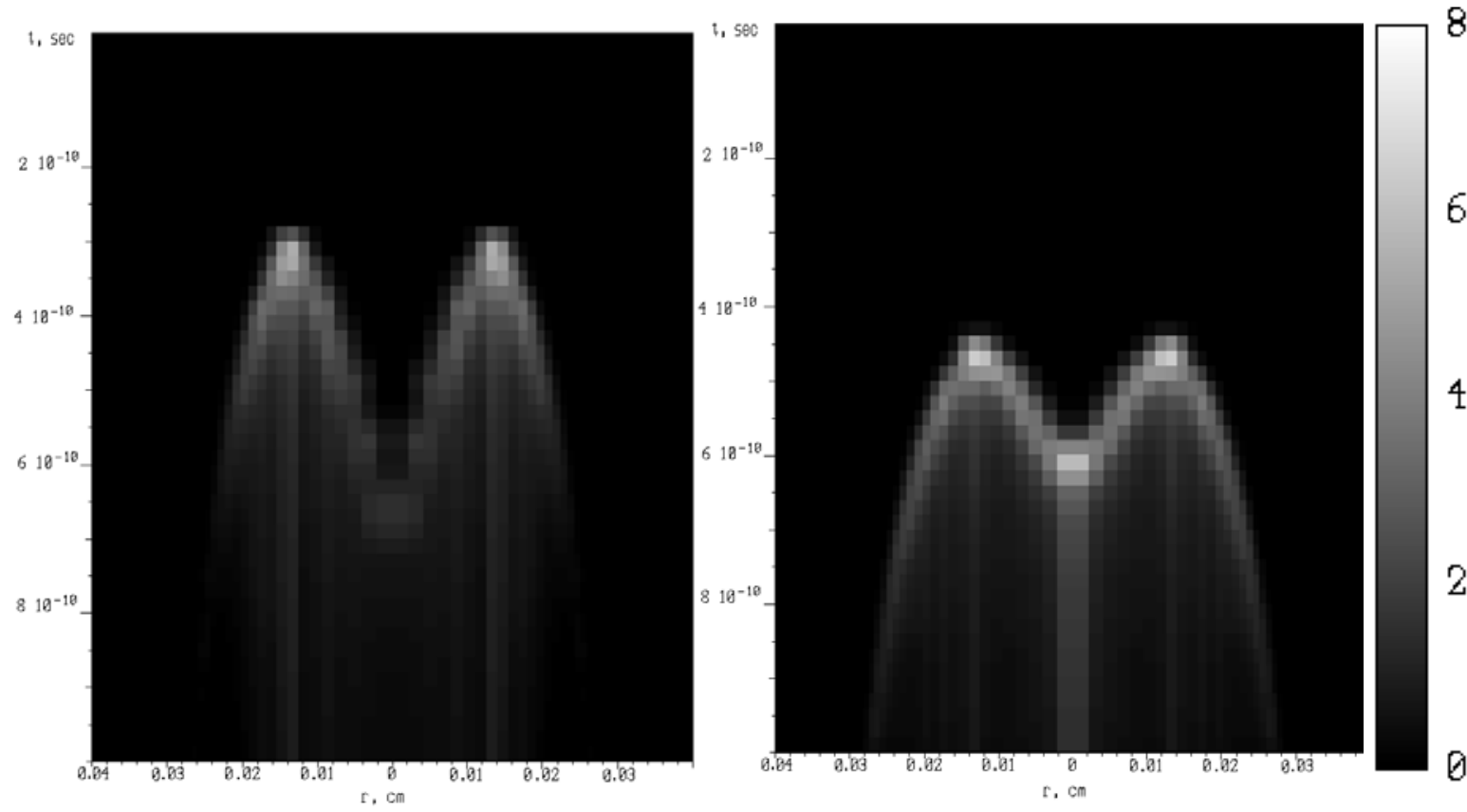

$8 \mathrm{~T}, \mathrm{eV}$

Fig. 5. The temperature of the rear side (the last cell of the target) as a function of time for the Al (left) and the Al-foam $\left(\rho_{\text {foam }}=50 \mathrm{mg} / \mathrm{cm}^{3}\right.$, right $)$ targets. The laser parameters are the same as in Fig. 2 . 

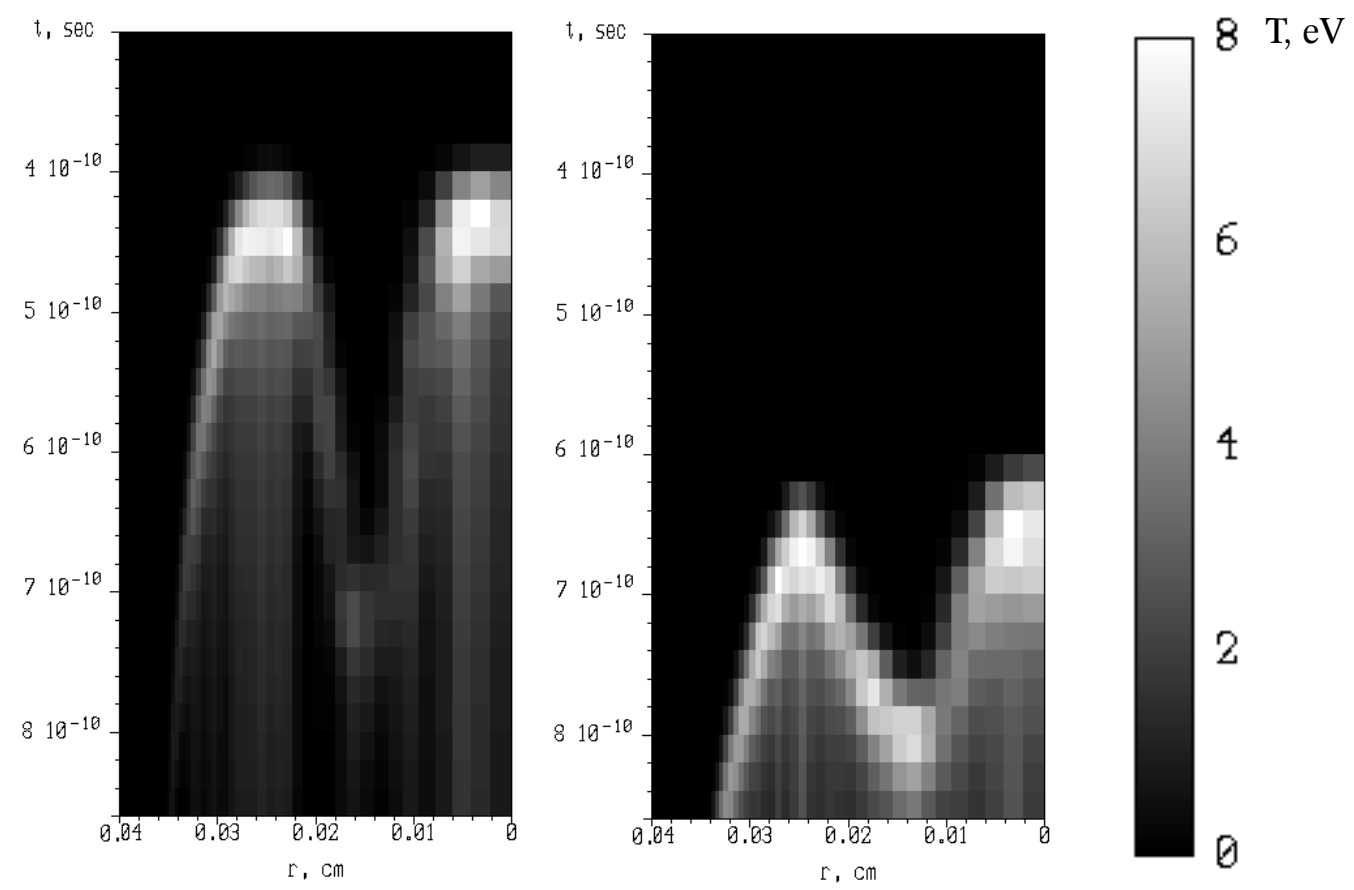

Fig. 6. The temperature of the rear side (the last cell of the target) as a function of time for the Al (left) and Al-foam $\left(\rho_{\text {foam }}=50 \mathrm{mg} / \mathrm{cm}^{3}\right.$, right $)$ targets. The laser parameters are the same as in Fig. 4.

the detector (streak camera) and the transmission optics. A direct comparison would require post-processing of the simulation data in order to obtain emissivity starting from temperature and density profiles in the target. However, we believe that this is not needed for a qualitative comparison. Indeed, although the total radiated power from target rear side approximately follows the blackbody Stefan-Boltzmann's law $\left(\approx \sigma T^{4}\right)$, the diagnostic spectral window is limited, due to the sensitivity of the streak camera and the optics transmission. Once we convolute the blackbody spectrum with the actual spectral sensitivity, we find that the dependence of the brightness on the surface temperature is close to linear for a quite large range of temperatures.

\section{Conclusion}

We have experimentally observed and numerically analyzed the behavior of shocks generated by a 'double laser spot' on foam-Al layered targets. We have observed the shock breakout on the target rear side and we saw that the region between the two spots becomes brighter that the spots themselves. This is explained as a result of the collision between the two individual shocks, generating larger pressure in the collision region. We performed hydrodynamic simulations in $2 \mathrm{D}$, which despite the assumption of axial symmetry seem to reproduce the qualitative feature of our results.

It is interesting to compare the present results with those obtained in Ref. [16], where large-scale non-uniformities were smoothed by placing a gas jets before the $\mathrm{Al}$ target. In that case, the 'gas layer' was undercritical and the laser beam was smoothed as it was propagating through it. The laser was directly interacting at the front of the Al surface, no shock was created in the gas layer and therefore there was no impedance mismatch effect, which is one of the main effects reported in the present work, and the effect of collision of the reflected shocks did not take place there.

Acknowledgments. We warmly acknowledge technical staff of PALS. A.A. is also grateful to ESF (program SILMI), COST (STSM visit grant in the framework of COST Action MP1208 "Developing the physics and the scientific community for inertial fusion"), RFBR (12-01-96500, 12-01-96501), and Dynasty Foundation of D. Zimin for support.

\section{References}

1. Stevenson, R. M., Pepler, D. A., Danson, C. N., Norman, M. J., Bett, T. H., \& Ross, I. N. (1994). Binary-phase zone plate arrays for the generation of uniform focal profiles. Opt. Lett., 19(6), 363-365.

2. Koenig, M., Faral, B., Boudenne, J. M., Batani, D., Benuzzi, A., \& Bossi, S. (1994). Optical smoothing techniques for shock wave generation in laser-produced plasmas. Phys. Rev. E, 50(5), R3314.

3. Batani, D., Bleu, C., \& Lower, Th. (2002). Design, simulation and application of phase plates. Eur. Phys. J. D, 19, 231-243.

4. Kato, Y., Mima, K., Miyanaga, N., Arinaga, S., Kitagawa, Y., Nakatsuka, M., \& Yamanaka, C. (1984). Random phasing of high-power lasers for uniform target acceleration and plasma-instability suppression. Phys. Rev. Lett., 53(11), 1057.

5. Dixit, S. N., Lawson, J. K., Manes, K. R., Powell, H. T., \& Nugent, A. (1994). Kinoform phase plates for focal plane irradiance profile control. Opt. Lett., 19(6), 417-419.

6. Skupsky, S., Short, R. W., \& Kessler, T. (1989). Improved laser-beam uniformity using the angular 
dispersion of frequency modulated light. J. Appl. Phys., 66, 3456.

7. Lehmberg, R. H., \& Obenschain, S. P. (1983). Use of induced spatial incoherence for uniform illumination of laser fusion targets. Opt. Commun., 46, 27-31.

8. Willi, O., Afshar-rad, T., Coe, S., \& Giulietti, A. (1990). Study of instabilities in long scale-length plasmas with and without laser-beam-smoothing techniques. Phys. Fluids, 2, 1318-1324.

9. Batani, D., Bossi, S., Benuzzi, A., Koenig, M., Faral, B., Boudenne, J. M., Grandjouan, N., Atzeni, S., \& Temporalet, M. (1996). Optical smoothing for shock-wave generation: application to the measurement of equations of state. Laser Part. Beams, 14(2), 211-223.

10. Montgomery, D. S., Moody, J. D., Baldis, H. A., Afeyan, B. B., Berger, R. L., Estabrook, K. G., Lasinski, B. F., Williams, E. A., \& Labaune, C. (1996). Effects of laser beam smoothing on stimulated Raman scattering in exploding foil plasmas. Phys. Plasmas, 3(5), 1728. http://dx.doi.org/10.1063/1.871682.

11. Labaune, C., Baldis, H. A., Schifano, E., Bauer, B. S., Maximov, A., Ourdev, I., Rozmus, W., \& Pesme, D. (2000). Enhanced forward scattering in the case of two crossed laser beams interacting with a plasma. Phys. Rev. Lett., 85(8), 1658.

12. Emery, M. H., Gardner, J. H., Lehmberg, R. H., \& Obenschain, S. P. (1991). Hydrodynamic target response to an induced spatial incoherence-smoothed laser beam. Phys. Fluids B, 3, 2640-2650.

13. Desselberger, M., Afshar-rad, T., Khattak, F., Viana, S., \& Willi, O. (1992) Nonuniformity imprint on the ablation surface of laser-irradiated targets. Phys. Rev. Lett., 68(10), 1539.

14. Batani, D., Balducci, A., Nazarov, W., Löwer, Th., Hall, T., Koenig, M., Faral, B., Benuzzi, A., \& Temporal, M. (2001). Use of low-density foams as pressure amplifiers in equation-of-state experiments with laser-driven shock waves. Phys. Rev. E, 63(4), 046410.
15. Batani, D., Nazarov, W., Hall, T., Löwer, Th., Koenig, M., Faral, B., Benuzzi-Mounaix, A., \& Grandjouan, N. (2000). Foam smoothing studied through laser produced shocks. Phys. Rev. E, 62(6), 8573-8582.

16. Benocci, R., Batani, D., Dezulian, R., Redaelli, R., Lucchini, G., Canova, F., Stabile, H., Faure, J. Krousky, E., Masek, K., Pfeifer, M., Skala, J., Dudzak, R., Koenig, M., Tikhonchuk, V., Nicolaï, Ph., \& Malka, V. (2009). Direct evidence of gas-induced laser beam smoothing in the interaction, with thin foils. Phys. Plasmas, 16(1), 012703. http://dx.doi. org/10.1063/1.3056396.

17. Jungwirth, K., Cejnarova, A., Juha, L., Kralikova, B., Krasa, J., Krousky, E., Krupickova, P., Laska, L., Masek, K., Mocek, T., Pfeifer, M., Präg, A., Renner, O., Rohlena, K., Rus, B., Skala, J., Straka, P., \& Ullschmied, J. (2001). The Prague Asterix Laser System. Phys. Plasmas, 8, 2495. http://dx.doi. org/10.1063/1.1350569.

18. Zel'dovich, Ya. B., \& Raizer, Yu. P. (2002). Physics of shock waves and high-temperature hydrodynamical phenomena. Dover, New York.

19. Lindl, J. (1995). Development of indirect-drive approach to inertial confinement fusion and target physics basis for ignition and gain. Phys. Plasmas, 2, 3933-4024.

20. Ramis, R., Meyer-ter-Vehn, J., \& Ramírez, J. (2009). MULTI2D - a computer code for two-dimensional radiation hydrodynamics. Comput. Phys. Commun., 180, 977-994.

21. Aliverdiev, A., Batani, D., Dezulian, R., Vinci, T., Benuzzi-Mounaix, A., Koenig, M., \& Malka, V. (2008). Hydrodynamics of laser-produced plasma corona by optical interferometry. Plasma Phys. Control. Fusion, 50, 105013.

22. Aliverdiev, A., Batani, D., Antonelli, L., Jakubowska, K., Dezulian, R., Amirova, A., Gajiev, G., Khan, M., \& Pant, H. C. (2014). Use of multilayer targets for achieving off-Hugoniot states. Phys. Rev. E, 89, 053101. 